\title{
ON QUASI-DUO RINGS
}

\author{
by HUA-PING YU
}

(Received 12 March, 1993; revised 8 June, 1993)

1. Introduction. Bass [1] proved that if $R$ is a left perfect ring, then $R$ contains no infinite sets of orthogonal idempotents and every nonzero left $R$-module has a maximal submodule, and asked if this property characterizes left perfect rings ([1], Remark (ii), p. 470). The fact that this is true for commutative rings was proved by Hamsher [12], and that this is not true in general was demonstrated by examples of Cozzens [7] and Koifman [14]. Hamsher's result for commutative rings has been extended to some noncommutative rings. Call a ring left duo if every left ideal is two-sided; Chandran [5] proved that Bass' conjecture is true for left duo rings. Call a ring $R$ weakly left duo if for every $r \in R$, there exists a natural number $n(r)$ (depending on $r$ ) such that the principal left ideal $R r^{n(r)}$ is two-sided. Recently, Xue [21] proved that Bass' conjecture is still true for weakly left duo rings.

In Section 3 of this paper, we first generalize the above results to left quasi-duo rings. We call a ring $R$ a left (right) quasi-duo ring if every maximal left (right) ideal of $R$ is two-sided. It is shown that the class of all weakly left duo rings is properly contained in the class of all left quasi-duo rings but that several basic properties of weakly left duo rings as proved in [22] are valid for left quasi-duo rings. This is the content of Section 2. Furthermore, we establish in Section 4 the equivalence of the following conditions on a left quasi-duo ring $R$ : (1) $R$ is a left P-exchange ring; (2) the left free module ${ }_{R} R^{(N)}$ has finite exchange property; (3) $R / J(R)$ is von Neumann regular and $J(R)$ is left T-nilpotent; (4) Every left $R$-module has a maximal submodule. This generalizes one of the main results of Stock [19] (Theorem 4.8, p. 447). Examples are included to demonstrate that all generalizations are nontrivial.

Throughout this paper all rings are associative with identity and modules are unitary left modules unless otherwise specified. $J(R)$ always denotes the Jacobson radical of a ring $R$. Homomorphisms of modules will be written on the side of their arguments opposite to scalars.

2. Basic properties. Recall that a ring $R$ is called left (right) quasi-duo, if every maximal left (right) ideal of $R$ is two-sided. We immediately observe that every factor ring $R / I$ of a left quasi-duo ring $R$ is again left quasi-duo. It is obvious that if $R$ is a field, or a division ring or a commutative ring and $n>1$ then the ring of upper triangular $n \times n$ matrices over $R$ is left quasi-duo. More generally, we have the following

Proposition 2.1. A ring $R$ is left (right) quasi-duo if and only if every upper triangular matrix ring (finite or infinite) $U T M_{n \times n}(R)$ is left (right) quasi-duo if and only if every lower triangular matrix ring (finite or infinite) $L T M_{n \times n}(R)$ is left (right) quasi-duo, where $n$ is allowed to be any finite or infinite cardinal number.

Proof. Let $S=U T M_{n \times n}(R)$. For simplicity, we only prove the upper triangular countably infinite case, the proof of other cases are similar.

Glasgow Math. J. 37 (1995) 21-31. 
One can check that

$$
J(S)=\left(\begin{array}{ccccc}
J(R) & R & R & R & \ldots \\
0 & J(R) & R & R & \cdots \\
0 & 0 & J(R) & R & \ldots \\
0 & 0 & 0 & J(R) & \ldots \\
\cdots & \ldots & \ldots & \cdots & \ldots
\end{array}\right) .
$$

It is easy to check that a ring $R$ is left quasi-duo if and only if $R / J(R)$ is left quasi-duo, so we need only show that

$$
S / J(S)=\left(\begin{array}{cccc}
R / J(R) & 0 & 0 & \ldots \\
0 & R / J(R) & 0 & \ldots \\
0 & 0 & R / J(R) & \ldots \\
\ldots & \ldots & \ldots & \ldots
\end{array}\right)
$$

is left quasi-duo. But this is clear, since $S / J(S)$ is just the direct product of countably many copies of $R / J(R)$, and it is straightforward that the product ring $\prod_{i \in I} R_{i}$ is left quasi-duo if and only if each $R_{i}$ is left quasi-duo.

Recall that a ring $R$ is called weakly left duo if for every $r \in R$, there exists a natural number $n(r)$ (depending on $r$ ) such that the principal left ideal $R r^{n(r)}$ is two-sided. Yao [22] proved that weakly left duo rings are normal (i.e. idempotents are central), which extends the older result of Courter [6] (Theorem 1.3) for left or right duo rings. Therefore, the class of weakly left duo rings is properly contained in the class of left quasi-duo rings by our Proposition 2.1 and Proposition 2.2, since upper triangular matrix rings have non-central idempotents.

Proposition 2.2. Every weakly left (right) duo ring is left (right) quasi-duo.

Proof. Let $R$ be a weakly left duo ring. Take any maximal left ideal ${ }_{R} M$, we want to show that $M x \subset M$ for all $x \in R$.

If not, $M x+M=R$ by maximality of $M$. Then we have

$$
m x+m^{\prime}=1
$$

for some $m \in M, m^{\prime} \in M$. Choose a natural number $t$ such that $R(x m)^{\prime}$ is two-sided, thus

$$
\left(1+(m x)+\ldots+(m x)^{t}\right) m^{\prime}=1-(m x)^{t+1}=1-m \cdot(x m)^{t} \cdot x .
$$

The left side is in $M$ because $m^{\prime}$ is, and $(m x)^{t+1} \in M$ because $x m$ is and $R(x m)^{t}$ is two-sided. This implies $1 \in M$, a contradiction.

The next result, although very straightforward, is a key one in our investigation.

LeMma 2.3 For left or right quasi-duo ring $R$, all nilpotent elements are in $J(R)$.

Proof. Put $P={ }_{R} R$ and $S=$ End $P$. Consider $P$ as a projective module over $R$. It is well-known that $J(S)=\{a \in S \mid \operatorname{Im} a \ll P\}$. 
Suppose $a \in S$ and $a^{n}=0$, let

$$
P a+N=P
$$

where ${ }_{R} N \leq{ }_{R} P$. We want to show that $N=P$, which implies that $a \in J(S)$. Since the union of every ascending chain of proper submodules of $P$ is proper, by a Zorn's lemma argument, we may assume that ${ }_{R} N$ is a maximal submodule of ${ }_{R} P$. Applying $a$ to both sides of the equality, we have

$$
P a^{2}+N a=P a
$$

so

$$
P a^{2}+N a+N=P \text {. }
$$

Continuing in this way, we end up with

$$
N a^{n-1}+\ldots+N a^{2}+N a+N=P .
$$

Since $R$ is left quasi-duo, we get $N=P$ as desired.

Yue Chi Ming [23] proved that if $R$ is weakly left duo and $J(R)=0$, then $R$ is reduced (i.e. contains no nonzero nilpotent elements). This is also true for left quasi-duo rings.

COROLlary 2.4 If $R$ is left (or right) quasi-duo and $J(R)=0$, then $R$ is reduced.

Due to the left-right symmetry in the above Lemma and Corollaries, every conclusion in this paper concerning right quasi-duo rings has an obvious "left" version. From now on, we will confine ourselves to right quasi-duo rings.

Let $B M(R)$ denote the Brown-McCoy radical of the ring $R$. Recall that $B M(R)$ is just the intersection of all maximal two-sided ideals of $R$. Yao [22] proved that right primitive, weakly right duo rings are division rings [22, Theorem 1], that for a weakly right duo ring $R, J(R)=B M(R)[22$, Corollary 2], and that semiprimitive weakly right duo rings are subdirect products of division rings [22, Corollary 1]. All these are true for right quasi-duo rings, and are even easier to prove. Namely, let $R$ be a right quasi-duo ring, then: (1) if $R$ is right primitive, then $R$ is a division ring; (2) if $R$ is simple, then $R$ is a division ring; (3) $J(R)=B M(R) ;(4)$ if $J(R)=0$, then $R$ is a subdirect product of division rings.

Our next goal, Theorem 2.5 and Theorem 2.6, is to generalize and sharpen Theorem 2 of [22], which says that for a weakly right duo ring $R$, if every prime ideal is maximal, then $R$ is $\pi$-regular and Lemma 5 of [22], which says that for a weakly right duo ring $R$, if the prime radical $P(R)=0$ and every prime ideal of $R$ is maximal, then $R$ is $\pi$-regular. A ring $R$ is called strongly regular if for every $a \in R$, there exists $b \in R$ such that $a^{2} b=a$. Note that strong regularity implies regularity, which in turn, implies $\pi$-regularity, but none of the converse implications holds in general. Call a ring $R$ strongly $\pi$-regular if the descending chain $R a \supseteq R a^{2} \supseteq R a^{3} \supseteq \ldots$ terminates. It is well known that this definition is left-right symmetric [8] and that strongly $\pi$-regular rings are properly contained in the class of $\pi$-regular rings [10].

THEOREM 2.5 Let $R$ be a right quasi-duo ring. If every prime ideal of $R$ is right primitive (weaker than maximal), then $R$ is strongly $\pi$-regular and $R / J(R)$ is strongly regular.

Proof. Fisher and Snider [10] proved that a ring $R$ is strongly $\pi$-regular if and only if $R / Q$ is strongly $\pi$-regular for every prime ideal $Q$ of $R$. Assuming that primes are 
primitive, we have that $R / Q$ is a division ring for every prime ideal $Q$ of $R$, since primitive quasi-duo rings are division rings by definition. Therefore $R$ is strongly $\pi$-regular. Since $R / J(R)$ is reduced by Corollary $2.4, R / J(R)$ is strongly regular.

THEOREM 2.6 Let $R$ be a right quasi-duo ring. Then the following are equivalent:

(1) the prime radical $P(R)=0$ and every prime ideal of $R$ is maximal,

(2) the prime radical $P(R)=0$ and every prime ideal of $R$ is right primitive,

(3) $R$ is strongly regular.

Proof. (1) $\Rightarrow$ (2): Trivial.

(2) $\Rightarrow$ (3): Since every prime ideal is right primitive, $P(R)=J(R)=0$. The rest of the proof follows from Theorem 2.5.

(3) $\Rightarrow(1)$ : Let $Q$ be a prime ideal of $R$, then $R / Q$ is a division ring by Theorem 3.2 of [11]. So $Q$ is maximal and $P(R)=0$ follows from the regularity of $R$.

The rest of this section concerns a problem of Fisher [9]. Recall that a ring $R$ is called fully left (right) idempotent if every left (right) ideal ${ }_{R} I \leq{ }_{R} R\left(I_{R} \leq R_{R}\right)$ is idempotent: $I^{2}=I$, and that $R$ is a left (right) $V$-ring if every left (right) simple $R$-module is injective. One of the three problems considered in [9] asked: for what rings $R$ is it true that $R$ is regular if and only if $R$ is a V-ring?

Armendariz and Fisher proved that for PI-rings (rings satisfying a polynomial identity) the above question has an affirmative answer [9, Theorem 16]. Recently, Yue Chi Ming [23, Proposition 7] proved an analogous result for weakly right duo rings, extending an older result of Chandran for right duo rings [5, Theorem 1]. We will show here that a similar result also holds for left (right) quasi-duo rings. Moreover, we prove that over a left (right) quasi-duo ring $R$, if the group ring $R[G]$ is a left or right V-ring, then it is regular. When the group $G$ is finite, the converse also holds. These again extend the results of [21, Proposition 2 and Corollary 2, p. 216].

The equivalences of (2), (3) and (5) in the following theorem have been proved in Brown [2, Theorem 4.11, p. 700].

THEOREM 2.7. For a right quasi-duo ring $R$, the following are equivalent:

(1) $R$ is von Neumann regular;

(2) $R$ is strongly regular;

(3) $R$ is regular and duo (i.e. left duo and right duo);

(4) $R$ is a left $V$-ring;

(5) $R$ is a right $V$-ring;

(6) $R$ is fully left idempotent;

(7) $R$ is fully right idempotent.

Proof. (1) $\Rightarrow$ (2): Trivial by Corollary 2.4 .

$(2) \Rightarrow(3)$ : Actually, (2) and (3) are equivalent for arbitrary rings. see, for example, [18, Proposition 1.2.3, p. 40].

(3) $\Rightarrow(5)$ : Brown $[2$, Theorem 4.8, p. 700$]$ showed that for a right duo ring $R, R$ is regular if and only if $R$ is a right $\mathrm{V}$-ring.

$(5) \Rightarrow(7)$ : Right V-rings are fully right idempotent by Fisher [9, Corollary 7, p. 105]. 
(7) $\Rightarrow(1)$ : Proposition 14 (1) of Ramamurthi [17] proved that the Jacobson radical of any fully right idempotent ring is zero, so $J(R)=0$. Then $R$ is reduced by Corollary 2.4. To show that $R$ is regular, it suffices to show that each factor ing $\bar{R}$ of $R$ which contains no nonzero divisor is a division ring, this is by Goodearl [11, Theorem 1.21]. Let $\bar{R}$ be such a factor ring of $R . \bar{R}$ is again fully right idempotent by [17, Proposition 5]. Since $\vec{R}$ contains no nonzero divisor, $\bar{R}$ is simple by [17, Proposition 7]. Then it follows that $\bar{R}$ is a division ring.

The other implications follow from symmetry.

Let $R$ be a ring and $G$ a group (not necessarily finite). It is well known that the group ring $R[G]$ is von Neumann regular if and only if: (1) $R$ is regular, (2) $G$ is locally finite (every finitely generated subgroup is finite), (3) the order of every element of $G$ is a unit in $R$. (see, for example, [15, p. 155]). Similarly, $R[G]$ is fully left (right) idempotent if and only if: (1) $R$ is fully left (right) idempotent, (2) $G$ is locally finite, (3) the order of each element of $G$ is a unit in $R$ [9, Theorem 9]. For a "weakly left duo" version of the next two results, see [21, p. 216].

Proposition 2.8. Let $R$ be a left quasi-duo ring and $G$ a group, the following statements are equivalent:

(1) $R[G]$ is regular;

(2) $R[G]$ is fully left idempotent;

(3) $R[G]$ is fully right idempotent.

Proof. Straightforward by Theorem 2.7 and the above mentioned results concerning the group ring $R[G]$.

COROLLARY 2.9. Let $R$ be a left quasi-duo ring and $G$ a group.

(1) If $R[G]$ is a left or right $V$-ring, then $R[G]$ is regular.

(2) If $G$ is finite, then $R[G]$ is a left $V$-ring if and only if it is regular if and only if it is a right $V$-ring.

Proof. (1) Suppose $R[G]$ is a left V-ring, then $R$ is a left V-ring, $G$ is locally finite and order of each element of $G$ is a unit in $R$ by Theorem 10 of [9]. So $R$ is regular by Theorem 2.7. Therefore $R[G]$ is regular by the above mentioned result.

(2) By Theorem 11 of [9], when $G$ is finite, $R[G]$ is a left V-ring if and only if $R$ is a left V-ring, $G$ is locally finite and order of each element of $G$ is a unit in $R$. Again the conclusion follows from Theorem 2.7.

For $G$ infinite, Farkas and Snider constructed an example in which $R[G]$ is regular but not a V-ring on either side (see Example 3 of [9], p. 109).

3. Bass' conjecture. Bass [1] proved that a left perfect $\operatorname{ring} R$ has the property that every left $R$-module has a maximal submodule and $R$ contains no infinite set of orthogonal idempotents, and asked if the converse holds also. In this section, we will establish the above converse for left (or right) quasi-duo rings. We start with an elementary observation. Let $R$ be a ring, $I_{1}$ and $I_{2}$ are two-sided ideals of $R$. If ${ }_{R}\left(R / I_{1}\right) \cong_{R}\left(R / I_{2}\right)$ as left $R$-modules, then $I_{1}=I_{2}$.

For a "weakly left duo" version of the following two lemmas, see Xue [21, Lemma 1 and Lemma 2, p. 212-213]. 
Lemma 3.1. Let $R$ be a left quasi-duo ring. If every left $R$-module has a maximal submodule, then every element of $R$ which is not a right zero divisor is a unit in $R$.

Proof. Let $x$ be an element of $R$ which is not a right zero divisor. Consider the left $R$-module

$$
{ }_{R} A=\bigoplus_{i=1}^{\infty} R y_{i}
$$

where $R y_{i} \cong R / R x^{i}$ (i.e. $\left.l_{R}\left(y_{i}\right)=R x^{i}\right)$. Let ${ }_{R} B=R y_{1}+\sum_{i=1}^{\infty} R\left(x y_{i+1}-y_{i}\right)$, then $A / B=\sum_{i=1}^{\infty} R \bar{y}_{i}$, with $\bar{y}_{i}=y_{i}+B$. Suppose $A \neq B$, then $A / B$ has a maximal submodule $M$. The relations $x \bar{y}_{i+1}=\bar{y}_{i}$ allow us to assume that

$$
\overline{y_{1}}, \ldots, \bar{y}_{i} \in M ; \bar{y}_{i+1}, \bar{y}_{i+2}, \ldots, \notin M
$$

Let ${ }_{R} T=A / B$; then $\overline{\bar{y}}_{i+1}=\bar{y}_{i+1}+M$ and $\overline{\bar{y}}_{i+2}=\bar{y}_{i+2}+M$ are nonzero elements of $T / M$ and $T / M$ is simple, so

$$
T / M=R \overline{\bar{y}}_{i+1}=R \overline{\bar{y}}_{i+2}
$$

Denote $l_{R}\left(\overline{\bar{y}}_{i+1}\right)$ and $l_{R}\left(\overline{\bar{y}}_{i+2}\right)$ by $I_{1}$ and $I_{2}$, respectively, then $I_{1}$ and $l_{2}$ are maximal left ideals of $R$ since $T / M$ is simple. Furthermore,

$$
R / I_{1} \cong R \overline{\bar{y}}_{i+1}=R \overline{\bar{y}}_{i+2} \cong R / I_{2}
$$

by the observation above, we have $I_{1}=I_{2}$, since $I_{1}, I_{2}$ are two-sided ideals of $R$. Note that $x \in I_{1}$, since $\bar{y}_{i} \in M$ and $x y_{i+1}-y_{i} \in B$ implying $x \bar{y}_{i+1}=\bar{y}_{i} \in M$, which is equivalent to $x \overline{\bar{y}}_{i+1}=\overline{\overline{0}}$. Then $x \in I_{2}$, which, in turn, gives us that $x \overline{\bar{y}}_{i+2}=\overline{\overline{0}}$, i.e. $x \bar{y}_{i+2}=\bar{y}_{i+1} \in M$, a contradiction. So we must have $A=B$.

We now suppose that

$$
y_{1}=\sum_{i=1}^{n} r_{i}\left(x y_{i+1}-y_{i}\right)=-r_{1} y_{1}+\sum_{i=2}^{n}\left(r_{i-1} x-r_{i}\right) y_{i}+r_{n} x y_{n+1}
$$

for some $r_{1}, r_{2}, \ldots, r_{n} \in R$. Since the $y_{i}$ are independent, we have

$$
y_{1}=-r_{1} y_{1} ; \quad r_{i-1} x-r_{i} \in l_{R}\left(y_{i}\right)=R x^{i}, \quad(i=2, \ldots, n) ; \quad r_{n} x \in l_{R}\left(y_{n+1}\right)=R x^{n+1} .
$$

But $x$ is not a right zero divisor, $r_{n} x \in R x^{n+1}$ implies $r_{n} \in R x^{n}$.

Suppose that $r_{k} \in R x^{k},(2 \leq k \leq n)$, then $r_{k-1} x-r_{k} \in R x^{k}$ implies $r_{k-1} x \in R x^{k}$. Again $r_{k-1} \in R x^{k-1}$.

Inductively we have $r_{1} \in R x$, which says that

$$
y_{1}=-r_{1} y_{1}=0 \text {. }
$$

So $R x=R$. Then $1=y x$ for some $y \in R$. But $(x y-1) x=0$, we have $x y=1$ since $x$ is not a right zero divisor. We have shown that $x$ is a unit as desired.

Lemma 3.2. Let $R$ be a left quasi-duo ring with $J(R)=0$ such that every left $R$-module has a maximal submodule, then $R$ is von Neumann regular.

Proof. It suffices to show that $R a \oplus l_{R}(a)=R$, for every $a \in R$. 
From the assumptions, $R$ is reduced by Corollary 2.4. In any reduced ring $l_{R}(a)=r_{R}(a)$, hence $l_{R}(a)$ is two-sided for every $a \in R$. Moreover, we claim that $R a \cap l_{R}(a)=0$. Take $x \in R a \cap l_{R}(a), x=y a$ for some $y \in R$ and $x a=0$. From this, $x y \in l_{R}(a)$, then $x^{2}=x y a=0$ implies $x=0$, since $R$ is reduced. For $0 \neq a \in R, \bar{R}=R / l_{R}(a)$ is a ring, since $l_{R}(a)$ is two-sided. Moreover, $\bar{R}$ is also a left quasi-duo ring and every left $\bar{R}$-module has a maximal submodule. Take any $\bar{r}=r+l_{R}(a) \in \bar{R} ;$ if $\bar{r} \cdot \bar{a}=\overline{0}$, then $r a \in R a \cap l_{R}(a)=0$, so $r \in l_{R}(a)$, i.e. $\bar{r}=\overline{0}$. Thus $\bar{a}$ is not a right zero divisor in $\bar{R}$. By Lemma $3.1, \bar{a}$ is a unit in $\bar{R}$. So $\bar{R} \bar{a}=\bar{R}$, which gives us

$$
R a \oplus l_{R}(a)=R .
$$

Therefore $R$ is regular.

We are now ready to prove the main result of this section.

THEOREM 3.3. For a left quasi-duo ring $R$, the following are equivalent:

(1) $R$ is left perfect;

(2) $R$ contains no infinite set of orthogonal idempotents and every left $R$-module has a maximal submodule.

Proof. (1) $\Rightarrow(2)$ : see Bass [1].

(2) $\Rightarrow(1)$ : Let $S=R / J(R)$. Since $J(R)$ is left T-nilpotent by Bass [1, Remark (ii), p. 470], it remains to show that $S$ is semisimple Artinian. It is well-known that countable orthogonal idempotents can be lifted modulo nil ideals, so $S$ cannot contain an infinite set of orthogonal idempotents. Let

$$
1=s_{1}+s_{2}+\ldots+s_{n}
$$

with the $s_{i}$ being pairwise orthogonal primitive idempotents. Since $S$ is left quasi-duo and $J(S)=0$, the $s_{i}$ are central idempotents by Corollary 2.4. Then $S$ has the following ring decomposition

$$
S=\bigoplus_{i=1}^{n} S s_{i}
$$

where each $S s_{i}$ is regular since $S$ is, by Lemma 3.2. But each $S s_{i}$ contains no nontrivial idempotents, so they are division rings.

Remark 3.4. Cozzens [7] and Koifman [14] constructed examples showing that Bass' conjecture is false in general. Both examples are simple non-division rings with Jacobson radical zero and contain no nontrivial idempotents, But all modules (left or right) over them do have maximal submodules (actually the rings they constructed are left and right V-rings, i.e. simple modules are all injective). Since simple quasi-duo rings are division rings, Cozzens and Koifman's examples are not quasi-duo on either side. This shows that the quasi-duo assumption is important.

4. P-exchange rings. Let $R$ be a left quasi-duo ring. Theorem 3.3 says that if every left $R$-module has a maximal submodule and $R$ contains no infinite set of orthogonal idempotents, then $R$ is left perfect. In this section, we consider the same problem with the condition on idempotents dropped. It turns out that left quasi-duo rings over which every left module has a maximal submodule are exactly those rings over which every projective 
left module has the exchange property, a property of modules introduced by Crawley and Jonsson [4]. A ring over which every projective left (right) module has the exchange property is called a left (right) P-exchange ring and has been studied recently by several authors (see, for example, Stock [19], Kambara and Oshiro [13]).

In [4], a module ${ }_{R} M$ is said to have the exchange property if for every module ${ }_{R} A$ and any two decompositions of ${ }_{R} A$

$$
{ }_{R} A=M^{\prime} \oplus N=\bigoplus_{i \in I} A_{i}
$$

where ${ }_{R} M^{\prime} \cong{ }_{R} M$, there exist submodules ${ }_{R} A_{i}^{\prime} \subset_{R} A_{i}$, such that

$$
A=M^{\prime} \oplus\left(\bigoplus_{i \in I} A_{i}^{\prime}\right)
$$

A module ${ }_{R} M$ is said to have the finite exchange property if the above condition is satisfied whenever the index set is finite. The importance of this notion lies in the fact that if the modules ${ }_{R} A_{i}(i \in I)$ are countably generated and all have the exchange property, then any two direct sum decompositions of ${ }_{R} A=\sum_{i \in I} A_{i}$ have isomorphic refinements (Crawley and Jonsson [4, Theorem 7.1]).

Warfield [20] called a ring $R$ an exchange ring if ${ }_{R} R$ has the exchange property, and proved that a module ${ }_{R} M$ has the exchange property if and only if End ${ }_{R} M$ is an exchange ring. Nicholson [16] called a ring $R$ a clean ring if every element of $R$ is a sum of a unit plus an idempotent, and proved that clean rings are exchange and the converse holds when all idempotents are central. Our first result in this section extends this to left (or right) quasi-duo rings. Since exchange rings with all idempotents central are left and right quasi-duo, by Burgess and Stephenson [3, Theorem 3.1(ii) (b), p. 162], and we have examples (e.g. the rings of $n \times n$ upper triangular matrices over a division ring) showing that there exists left quasi-duo exchange rings with non-central idempotents, the generalization is nontrivial.

Proposition 4.1. For an exchange ring $R$ with $J(R)=0$, the following are equivalent:

(1) $R$ is left quasi-duo;

(2) $R$ is reduced;

(3) $R$ is normal (i.e. all idempotents are central).

Proof. (1) $\Rightarrow(2)$ : By Corollary 2.4 .

(2) $\Rightarrow(3)$ : This is true for an arbitrary ring.

(3) $\Rightarrow(1)$ : By Burgess and Stephenson [3], this is true even without assuming that $J(R)=0$.

THEOREM 4.2. For a left or right quasi-duo ring $R$, the following are equivalent:

(1) $R$ is an exchange ring;

(2) $R$ is a clean ring.

Proof. (2) $\Rightarrow(1)$ : Every clean ring is an exchange ring (see Nicholson [16, Proposition 1.8(i)]).

$(1) \Rightarrow(2)$ : It is known that a ring $R$ is clean if and only if $R / J(R)$ is clean and idempotents lift modulo $J(R)$ [16, p. 272]. Also, $R$ is exchange if and only if $R / J(R)$ is 
exchange and idempotents lift modulo $J(R)$ [16, Proposition 1.5]. So, without loss of generality, we may assume that $J(R)=0$. Now the proof follows from Proposition 4.1 above.

It is still an open question whether there exists an exchange ring which is not clean.

Stock [19] called a ring $R$ left $P$-exchange if every projective left $R$-module has the exchange property. Unlike the exchange ring case, the notion of a P-exchange ring is not left-right symmetric, although it is obvious that P-exchange rings are exchange rings. It remains open to determine the structure of P-exchange rings (see Kambara and Oshiro [13]). For a ring $R$ with all idempotents central, Stock [19] proved that $R$ is left P-exchange if and only if $R / J(R)$ is von Neumann regular and $J(R)$ is left T-nilpotent [19, Theorem 4.8]. Based on the results of the previous sections, we are able to characterise those left quasi-duo rings which are left P-exchange, which, in the view of the following proposition, generalizes the above result of Stock.

Proposition 4.3. Every P-exchange ring (left or right) with all idempotents central is left and right quasi-duo.

Proof. Since P-exchange rings are exchange rings, by Burgess and Stephenson [3, Theorem 3.1, (ii) (b)], they are left and right quasi-duo.

The rings of $n \times n$ upper triangular matrices over a division ring are also examples of left quasi-duo left P-exchange ring which has non-central idempotents.

We are now ready to prove the main theorem of this section.

THEOREM 4.4. For a left quasi-duo ring $R$, the following are equivalent:

(1) $R$ is a left $P$-exchange ring;

(2) the left free module ${ }_{R} R^{(N)}$ has the finite exchange property;

(3) $R / J(R)$ is von Neumann regular and $J(R)$ is left $T$-nilpotent;

(4) Every left $R$-module has a maximal submodule.

Proof. (1) $\Rightarrow(2)$ : Trivial by definition.

$(2) \Rightarrow(3): J(R)$ is left T-nilpotent by Stock [19, Theorem 4.1 (2), p. 444]. Since $J(R / J(R))=0$, and $R / J(R)$ is also left quasi-duo, then $R / J(R)$ is reduced by Corollary 2.4.

To prove the von Neumann regularity of a ring without nonzero nilpotent elements, it suffices to show that every factor ring of it which contains no nonzero zero divisors is a division ring (see, for example, Goodearl [11, Theorem 1.21]). Let $\bar{R}$ be such a factor ring of $R / J(R)$, then $\bar{R}_{\bar{R}} \bar{R}^{(N)}$ also has the finite exchange property by Stock [19, Proposition 4.1 (1), p. 444]. So $J(\bar{R})$ is left T-nilpotent. But $\bar{R}$ has no nonzero divisors, thus $J(\bar{R})=0 . \bar{R}$ is also an exchange ring, by Nicholson [16, Proposition 1.9, p. 272] $\bar{R} a$ contains a nonzero idempotent $e$, say, for every $0 \neq a \in \bar{R}$. Again the fact that $\bar{R}$ contains no nonzero zero divisor forces $e=1$, which implies $\bar{R} a=\bar{R}$. Then it is straightforward to check that $\bar{R}$ is a division ring.

(3) $\Rightarrow(4)$ : The fact that $R / J(R)$ is regular and left quasi-duo implies that $R / J(R)$ is strongly regular by Theorem 2.7 (2). Also by the same theorem, $R / J(R)$ is a left V-ring. A left $V$-ring has have the property that every left module over it has a maximal submodule (see, for example, Koifman [14, Prop. 2.4]). But the fact that every left $R$-module has a maximal submodule is equivalent to every left $R / J(R)$-module having a maximal 
submodule and $J(R)$ being left T-nilpotent (see Hamsher [12, Lemma 1]), so we get the desired property of $R$.

(4) $\Rightarrow(3)$ : By [12, Lemma 1], $J(R)$ is left T-nilpotent and every $R / J(R)$-module has a maximal submodule. Since $R / J(R)$ is still left quasi-duo and $J(R / J(R))=0$, by Lemma $3.11, R / J(R)$ is regular.

(3) $\Rightarrow(1)$ : This is true even without the over all assumption on $R$ (see, for example, Stock [19, Theorem 4.2]).

The equivalence of (3) and (4) in Theorem 4.4 for commutative rings has been established by Hamsher [12, p. 1135]. Recently, Xue [21] proved that for weakly left duo rings, (3) and (4) are equivalent [21, Corollary 1]. Theorem 4.4 can also be viewed as a generalization of these results.

Of course, all generalizations we claimed here are nontrivial. For example, take $R$ to be the ring of $2 \times 2$ upper triangular matrices over a division ring $D$, then $R$ is left quasi-duo but not weakly left duo (nor weakly right duo). Moreover, $R$ is left perfect, so it serves as an example which is covered by our Theorem 3.3, but not by the analogous theorems of Xue [21, Theorem 3] and Chandran [5, Theorem 3]. Also, the same ring $R$ is an exchange ring and P-exchange ring, which is covered by our Theorems 4.2 and 4.4 but not by the analogous results of Nicholson [16, Proposition 1.8] and Stock [19, Theorem 4.8]. So, all the generalizations are nontrivial.

We conclude this paper by making one more negative observation on quasi-duo rings.

Remark 4.5. For any division ring $D$ (or more generally, any left or right quasi-duo ring $R$ ), the upper triangular matrix ring $S=U T M_{\infty \times \infty}(D)$ is always left quasi-duo by Proposition 2.1. But $J(S)=\left\{\left(a_{i j}\right) \in S \mid a_{i i}=0\right\}$ is not nil, hence not left T-nilpotent, $S$ can never be a P-exchange ring, or equivalently, a left max ring (i.e. every left module over it has a maximal submodule).

Acknowledgement. The author wishes to express his sincere gratitude to his Ph.D. advisor Prof. Vic Camillo for his encouragement and direction. The author also thanks the referee for many careful comments which have improved the presentation of this paper.

Added IN PRoof. (1) The open question whether there exists an exchange ring that is not clean (p. 29) has been settled in the negative by Camillo and Yu, Exchange rings, units and idempotents, Comm. Alg. 22 (1994), 4737-4749.

(2) The converse of Theorem 2.5 also holds. A strengthened version of Theorem 2.5 will be presented in Hua-Ping $\mathrm{Yu}$, On rings whose prime factors are simple (to appear).

\section{REFERENCES}

1. H. Bass, Finitistic dimension and a generalization of semiprimary rings, Trans. Amer. Math. Soc. 95 (1960), 466-488.

2. S. H. Brown, Rings over which every simple module is rationally complete, Canad. J. Math. 25 (1973), 693-701.

3. W. D. Burgess and W. Stephenson, Rings all of whose Pierce stalks are local, Canad. Math. Bull. 22 (1979), 159-164. 
4. P. Crawley and B. Jonsson, Refinements for infinite direct decompositions of algebraic systems, Pacific J. Math. 14 (1964), 797-855.

5. V. R. Chandran, On a conjecture of Hyman Bass, Pure Appl. Math. Sci. 4 (1976), 125-131.

6. R. C. Courter, Finite dimensional right duo algebras are duo, Proc. Amer. Math. Soc. 84 (1982), 157-161.

7. J. H. Cozzens, Homological properties of the ring of differential polynomials, Bull. Amer. Math. Soc. 76 (1970), 75-79.

8. M. F. Dischinger, Sur les anneaux fortement $\pi$-reguliers, C. R. Acad. Sci. Paris, Ser. A 283 (1976), 571-573.

9. J. W. Fisher, Von Neumann regular rings versus V-rings, Ring Theory, Marcel Dekker, 1974, 101-119.

10. J. W. Fisher and R. L. Snider, On the von Neumann regularity of rings with regular prime factor rings, Pacific J. Math. 54 (1974), 135-144.

11. K. R. Goodearl, Von Neumann Regular Rings (Pitman, 1979).

12. R. M. Hamsher, Commutative rings over which every module has a maximal submodule, Proc. Amer. Math. Soc. 18 (1967), 1133-1137.

13. H. Kambara and K. Oshiro, On P-exchange rings, Osaka J. Math. 25 (1988), 833-842.

14. L. A. Koifman, Rings over which every module has a maximal submodule, Math. Notes 7 (1970), 215-219.

15. J. Lambek, Lectures on Rings and Modules (Chelsea, 1976).

16. W. K. Nicholson, Lifting idempotents and exchange rings, Trans. Amer. Math. Soc. 229 (1977), 269-278.

17. V. S. Ramamurthi, Weakly regular rings, Canad. Math. Bull. 16 (1973), 317-321.

18. Bo Stenstrom, Rings of Quotients (Springer-Verlag, 1975).

19. J. Srock, On rings whose projective modules have exchange property, J. Algebra 103 (1986), 437-453.

20. R. B. Warfield, Exchange rings and decompositions of modules, Math. Ann. 199 (1972), 31-36.

21. W. Xue, On weakly right duo rings, Riv. Mat. Univ. Parma 15 (1989), 211-217.

22. X. Yao, Weakly right duo rings, Pure Appl. Math. Sci. 21 (1985), 19-24.

23. R. Yue Chi Ming, On annihilator ideals (IV), Riv. Mat. Univ. Parma 13 (1987), 19-27.

Department of Mathematics

THE UNIVERSITY OF IOWA

IOWA CITY

IA 52242, U.S.A. 